\title{
Utility of IOLMaster 700 Swept-Source Optical Coherence Tomography in Detecting Macular Disease for Preoperative Cataract Surgery Patients
}

\author{
Jeffrey $M a$ iD $^{1,2}$ \\ Chintan Pathak' \\ Abhijit Ramaprasad' \\ Surendra Basti ${ }^{1}$ \\ 'Department of Ophthalmology, Feinberg \\ School of Medicine, Northwestern \\ University, Chicago, IL, USA; \\ ${ }^{2}$ Department of Ophthalmology, UC \\ Davis Eye Center, University of California \\ Davis, Sacramento, CA, USA
}

Correspondence: Surendra Basti Department of Ophthalmology, Feinberg School of Medicine, Northwestern University, 645 North Michigan Avenue, Suite 440, Chicago, IL, 606II, USA

Tel + I 3129088152

Fax + 3125038152

Email sbasti@northwestern.edu
Purpose: To assess the efficacy of IOLMaster 700 (IOLM) biometer swept-source optical coherence tomography (SS-OCT) in detecting macular pathology before cataract surgery and to compare IOLM SS-OCT characteristics of foveal pathology with a widely used spectraldomain OCT (SD-OCT) system.

Patients and Methods: Retrospective analysis of 1156 consecutive eyes with IOLMaster 700 SS-OCT undergoing cataract surgery from January to June 2017 was performed. Approximately a third of these eyes (327 eyes) also had a SD-OCT scan performed previously. A single reviewer assessed each SS-OCT scan and identified them as "normal" or "abnormal." SS-OCT sensitivity and specificity in identifying foveal pathology was assessed using findings on Spectralis SD-OCT scans as the gold standard.

Results: Of 327 eyes with both IOLM SS-OCT and Spectralis SD-OCT scans, 121 eyes $(37.0 \%)$ had abnormal SS-OCT scans. Of these 121 eyes, SD-OCT scans confirmed pathology in 104 eyes $(86.0 \%)$. Of the remaining 206 eyes graded to have normal SS-OCT scans, 84 eyes $(40.8 \%)$ had normal SD-OCT scans, and 122 eyes $(59.2 \%)$ had pathologic findings on SD-OCT scans. For each pathologic condition, subtle but definitive differences existed in the appearance of the IOLM SS-OCT and SD-OCT images.

Conclusion: Using a normal or abnormal Spectralis SD-OCT scan as confirmation of absence or presence of foveal pathology respectively, we found a high positive predictive value $(86.0 \%)$ of an abnormal IOLM SS-OCT scan and a high specificity $(83.2 \%)$ but low sensitivity $(46.0 \%)$ and negative predictive value $(40.8 \%)$ of a normal-appearing SS-OCT scan. These results suggest that an abnormal IOLM SS-OCT scan in an eye without known pathology is a strong indicator of an abnormal macula and should prompt further evaluation of the retina to identify pathology prior to cataract surgery. Importantly, IOLM SS-OCT scans do not detect all macular pathology and cannot be used as a screening test for identifying macular pathology.

Keywords: optical biometry, macular OCT, cataract surgery preoperative testing

\section{Introduction}

The number of people affected by cataracts in the United States is increasing, with over 50 million people projected to have cataracts by $2050 .^{1}$ Excellent uncorrected visual acuity has increasingly become the expectation of patients and surgeons following cataract surgery. The health of the retina is an important determinant of post-surgical visual outcome. Identifying macular pathology before cataract surgery is essential as it helps set expectations for visual outcome, identifies patients who may be poor candidates for multifocal 
intraocular lenses, and detects comorbidities such as macular edema that may affect pre-operative and postoperative management.

Optical coherence tomography (OCT) is a highly effective imaging modality for detecting subtle macular abnormalities that may be difficult to visualize on fundoscopy. Several studies have demonstrated that OCT is more sensitive than slit lamp biomicroscopy in diagnosing macular diseases such as epiretinal membrane, macular degeneration, and cystoid macular edema in patients undergoing preoperative evaluation for cataract surgery. ${ }^{2,3}$ While there arguably is a role for routine preoperative OCT in all patients undergoing cataract surgery, such a practice raises logistical and financial challenges to a busy ophthalmology practice. In countries such as the United States, OCT is an insurance-reimbursable cost only in eyes with macular pathology. Hence, many cataract surgeons do not routinely obtain OCT imaging as part of their preoperative evaluation.

The IOLMaster 700 (IOLM) is a biometry device that performs a limited swept-source optical coherence tomography (SS-OCT) scan of the full length of the eye, allowing for identification of morphologic abnormalities such as crystalline lens tilt or decentration and measurement of the posterior corneal curvature. The SS-OCT scan of the retina is limited to the central $1.0-\mathrm{mm}$ of the fovea and is intended to assess patient fixation and consequently, accuracy of axial length measurements. Therefore, each biometry measurement taken with the IOLM has a limited SS-OCT available that surgeons can review and potentially use to identify foveal pathology prior to cataract surgery.

In this study, we assess the utility of the IOLM SSOCT in detecting foveal pathology in preoperative cataract surgery patients. We also characterize differences in appearance of common macular pathologic conditions on the IOLM SS-OCT compared with Spectralis spectraldomain optical coherence tomography (SD-OCT), a widely used SD-OCT platform.

\section{Patients and Methods}

This retrospective study performed at the Department of Ophthalmology, Northwestern University Feinberg School of Medicine, Chicago, USA was approved by the Institutional Review Board of Northwestern University and was conducted in accordance with the principles of the Declaration of Helsinki. The informed consent requirement was waived due to the retrospective nature of the study. Patient data were kept confidential and anonymous.
All eyes undergoing cataract surgery at Northwestern Memorial Hospital from January 2017 to June 2017 with biometry conducted by the Zeiss IOLMaster 700 (Carl Zeiss Meditec AG, Jena, Thuringia, Germany) were included in the study (Table 1). The SS-OCT on the IOLMaster 700 uses a laser wavelength of $1055-\mathrm{nm}$, with an acquisition speed of 2000 amplitude scans (A-scans) per second and depth of penetration of 44-mm, providing 6 line scans with $22-\mu \mathrm{m}$ axial resolution. Eyes that also had Spectralis SD-OCT (Heidelberg Engineering, Heidelberg, Germany) performed within 3 months of IOLMaster biometry were identified from this cohort. The decision to obtain a preoperative Spectralis SD-OCT was based on the clinical judgment and individual preoperative workflow of each attending ophthalmologist. In most instances, an SD-OCT was obtained if there were signs of possible macular pathology on dilated fundoscopic exam. Prior to surgery, all patients had been evaluated by an attending ophthalmologist with documentation of best-corrected visual acuity and dilated fundus examination. Eyes may or may not have been dilated at the time of biometry acquisition and SD-OCT scan, depending on whether an attending ophthalmologist's preoperative workflow included obtaining measurements and OCT scans during the initial dilated exam

Table I Preoperative Patient Demographics and Ocular Characteristics

\begin{tabular}{|l|c|}
\hline Number of Patients & $\mathbf{5 7 8}$ ( I I 56 Eyes) \\
\hline Gender & $226(39.1 \%)$ \\
Male & $352(60.9 \%)$ \\
\hline Female & \\
\hline Preoperative visual acuity & $12.3 \%$ \\
$20 / 20-20 / 25$ & $29.8 \%$ \\
$20 / 30-20 / 40$ & $24.1 \%$ \\
$20 / 50-20 / 60$ & $11.1 \%$ \\
$20 / 70-20 / 80$ & $12.3 \%$ \\
$20 / 100-20 / 250$ & $4.7 \%$ \\
$20 / 300-20 / 600$ & $5.6 \%$ \\
Count fingers or worse & \\
\hline Abnormal fundoscopy findings & $47(4.1 \%)$ \\
Drusen (including extramacular) & $25(2.2 \%)$ \\
Poor view & $23(2.0 \%)$ \\
Retinal pigment epithelium mottling/ & \\
irregularity & $19(1.6 \%)$ \\
Epiretinal membrane & $12(1.0 \%)$ \\
Microaneurysms or retinal hemorrhages & $11(1.0 \%)$ \\
Vascular attenuation & $6(0.5 \%)$ \\
Macular hole & $37(3.2 \%)$ \\
Other & \\
\hline
\end{tabular}


or if patients were instructed to return at a later date for preoperative measurements. Eyes were excluded from the study if the SS-OCT scan was deemed to be of poor image quality by the masked reviewer (for example, if the scan appeared overly blurred without distinguishable retinal layers, typically due to dense cataracts or motion artifact). Eyes were also excluded if any surgery or procedure (such as intravitreal anti-vascular endothelial growth factor injection) had been performed between acquisition of SS-OCT and SDOCT scans. Eyes were not excluded due to poor foveal fixation if the image quality was deemed by the masked reviewer to be adequate, because absence of a normal foveal contour in the scan may also be seen in certain pathologic conditions such as epiretinal membrane.

A single reviewer who is an ophthalmologist with prior experience in interpreting IOLM SS-OCT scans and who was masked to patients' prior ophthalmic history independently assessed each IOLM SS-OCT scan and graded it as "normal" or "abnormal." Scans were graded as "normal" if it included a foveal pit with a normal contour and retinal layers without any irregularities (Figure 1). Scans were graded as "abnormal" otherwise. Spectralis SD-OCT scans, considered as the gold standard screening test for macular pathology in this study, were identified as "normal" or "abnormal" based on their attending ophthalmologist's interpretation of the scan as documented in the patient's chart.

For all IOLM SS-OCT scans graded as "normal," the corresponding Spectralis SD-OCT scan was reviewed to confirm either absence of macular pathology (SS-OCT scan thereby considered a "true negative") or presence of macular pathology (SS-OCT scan thereby considered a "false negative"). Likewise, for all IOLM SS-OCT scans graded as "abnormal," the corresponding Spectralis SD-OCT scan was reviewed to confirm presence of pathology (SS-OCT scan thereby considered a "true positive") or absence of pathology (SS-OCT scan thereby considered a "false positive").

The corresponding IOLM SS-OCT scan for all abnormal Spectralis SD-OCT scans was also retrospectively reevaluated to assess whether any abnormal morphological features were indeed present on the SS-OCT scan and whether the IOLM SS-OCT scan had been incorrectly graded as "normal" during the masked review. Statistical analysis was performed using Microsoft Excel 2011 (Microsoft, Inc., Redmond, WA, USA). Sensitivity was calculated as the ratio of number of true positives (abnormal SS-OCT scans with abnormal SD-OCT scans) to the total number of abnormal SD-OCT scans. Specificity was calculated as the ratio of number of true negatives (normal SSOCT scans with normal SD-OCT scans) to the total number of normal SD-OCT scans. Positive predictive value was calculated as the ratio of number of true positives to the total number of abnormal SS-OCT scans, and negative predictive value was calculated as the ratio of number of true negatives to the total number of normal SS-OCT scans.

\section{Results}

A total of 1156 eyes that underwent preoperative cataract surgery evaluation with IOLM SS-OCT scans were

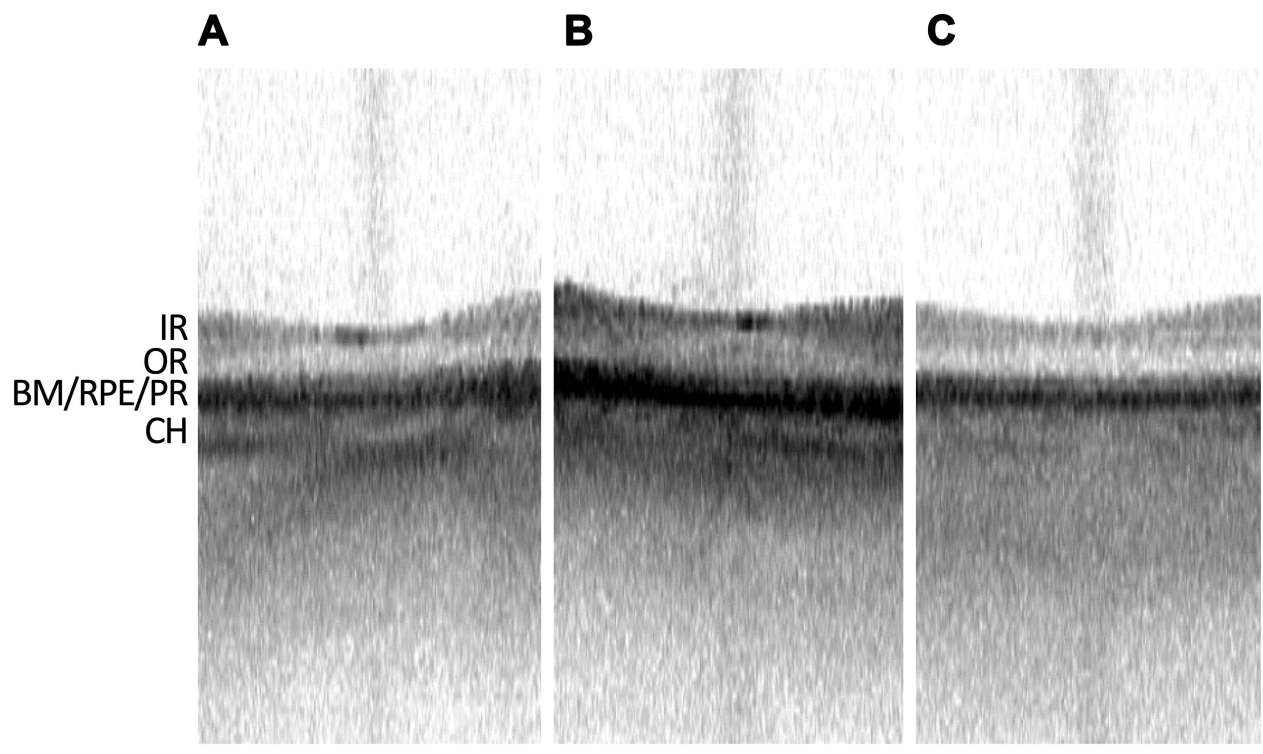

Figure I Representative swept-source OCT of normal foveas. (A-C) Representative IOLMaster 700 SS-OCT of three different non-pathological foveas, with inner retinal (IR) layers, outer retina (OR) layers, Bruch's Membrane (BM), retinal pigment epithelium (RPE), photoreceptor layer (PR), and choroid (CH) layer denoted. 
included in this study. Of 1156 SS-OCT scans included, 977 (84.5\%) were graded as "normal" (displaying a foveal pit with a normal contour and retinal layers without any irregularities) and $179(15.4 \%)$ were graded as "abnormal." Figure 1 shows representative IOLM SS-OCT scans of normal eyes without foveal pathology. In each, a foveal pit with a normal contour is present. While individual retinal layers cannot be clearly delineated due to the low resolution of the IOLM SS-OCT, roughly 4 distinct bands are identifiable in each SS-OCT scan (posterior to anterior): (1) choroid layer, (2) Bruch's membrane, retinal pigment epithelium, and photoreceptor layers, (3) outer retinal layers, and (4) inner retinal layers.

There were $327(28.3 \%)$ eyes in the study that had both an IOLM SS-OCT scan, as well as a Spectralis SD-OCT conducted within three months of the SS-OCT scan (Table 2). This cohort was used to assess the sensitivity and specificity of the IOLM SS-OCT scan in detecting foveal abnormalities. Of all 327 IOLM SS-OCT scans, $206(63.0 \%)$ scans were graded as "normal," and 121 $(37.0 \%)$ scans were graded as "abnormal." Of the 121 IOLM SS-OCT scans that were graded as "abnormal,"
Spectralis SD-OCT confirmed macular pathology in 104 (true positive rate of $86.0 \%$ ), while 17 (false positive rate of 14\%) abnormal SS-OCT scans had a corresponding normal Spectralis SD-OCT scan. Of the 104 eyes with an abnormal SS-OCT scan that was confirmed by Spectralis SD-OCT scan, the most common finding on the Spectralis SD-OCT scan was epiretinal membrane in $38(36.5 \%)$ scans, followed by drusen in $27(26.0 \%)$ scans, macular hole in $10(9.6 \%)$ scans, cystoid macular edema in 7 (6.7\%) scans and diabetic macular edema in $4(3.8 \%)$ scans. Thirty-one (29.8\%) scans showed various other conditions, such as retinal atrophy, degenerative myopia and pattern dystrophy on SD-OCT.

Of the 206 IOLM SS-OCT scans that were graded as "normal," Spectralis SD-OCT scan confirmed absence of macular pathology in 84 (true negative rate of $40.8 \%$ ) scans, while 122 (false negative rate of 59.2\%) scans had macular pathology on the corresponding Spectralis SDOCT scan.

Viewed from another vantage, of 226 eyes with an abnormal Spectralis SD-OCT scan, 104 (sensitivity of $46.0 \%$ ) had a SS-OCT scan that was correctly graded as

Table 2 Analysis of Efficacy of SS-OCT and SD-OCT in Detecting Macular Pathology and Most Common Macular Pathologies Identified

\begin{tabular}{|c|c|c|c|}
\hline \multicolumn{4}{|c|}{ Total eyes with both SS-OCT and SD-OCT $=327$} \\
\hline \multicolumn{4}{|l|}{ “Normal” SS-OCT = 206 eyes } \\
\hline \multicolumn{2}{|l|}{ Normal SD-OCT $=84$ eyes } & \multicolumn{2}{|l|}{ True negative $=40.8 \%$} \\
\hline \multicolumn{2}{|l|}{ Abnormal SD-OCT $=122$ eyes } & \multicolumn{2}{|l|}{ False negative $=59.2 \%$} \\
\hline \multicolumn{4}{|l|}{ "Abnormal" SS-OCT $=121$ eyes } \\
\hline \multicolumn{2}{|l|}{ Normal SD-OCT $=17$ eyes } & \multicolumn{2}{|l|}{ False positive $=14.0 \%$} \\
\hline \multicolumn{2}{|l|}{ Abnormal SD-OCT $=104$ eyes } & \multicolumn{2}{|l|}{ True positive $=86.0 \%$} \\
\hline & $\begin{array}{l}\text { Among eyes with abnormal SS- } \\
\text { OCT and SD-OCT (true positive, } \\
\text { I04 eyes) }\end{array}$ & $\begin{array}{l}\text { Among eyes with normal SS-OCT and } \\
\text { abnormal SD-OCT (false negative, } 122 \\
\text { eyes) }\end{array}$ & $\begin{array}{l}\text { Among all } \\
\text { abnormal SD- } \\
\text { OCT ( } 226 \text { eyes) }\end{array}$ \\
\hline Epiretinal membrane & $38(36.5 \%)$ & $33(27.0 \%)$ & $7 \mathrm{l}(3 \mathrm{I} .4 \%)$ \\
\hline Drusen & $27(26.0 \%)$ & $19(15.5 \%)$ & $46(20.4 \%)$ \\
\hline Macular hole & $10(9.6 \%)$ & $6(4.9 \%)$ & $16(7.1 \%)$ \\
\hline Cystoid macular edema & $7(6.7 \%)$ & $8(6.6 \%)$ & $15(6.6 \%)$ \\
\hline Diabetic macular edema & $4(3.8 \%)$ & $7(5.3 \%)$ & $11(4.9 \%)$ \\
\hline $\begin{array}{l}\text { Other (including non-central macular edema, } \\
\text { retinal dystrophy, degenerative myopia, } \\
\text { macular scars) }\end{array}$ & 31 (29.8\%) & 49 (4I.0\%) & $80(35.4 \%)$ \\
\hline
\end{tabular}


“abnormal" and $122(54.0 \%)$ had a SS-OCT scan that was incorrectly graded as "normal."

Epiretinal membranes, the most common macular pathology among cataract surgery patients in this study, have a subtle appearance on the IOLM SS-OCT (Figure 2). While the membrane itself may be difficult to discern (in contrast to its hyperreflective linear appearance in SD-OCT scans), the appearance of a thickened fovea with straightening or flattening of the foveal pit should be suggestive of an epiretinal membrane and hyporeflective intraretinal cysts may be present as well.

Macular edema is easily visualized on SS-OCT and appears as a thickened fovea with hyporeflective intraretinal or subretinal spaces (Figure 3). Differentiating between intraretinal and subretinal fluid is possible on SSOCT.

Drusen are another common finding on SS-OCT and appear as elevated deformations of the RPE layer (Figure 4). Large drusen or pigment epithelial detachments are easily identifiable beneath this band but smaller drusen have a more subtle appearance and may be difficult to discern on SS-OCT.

Macular holes are also easy to identify on SS-OCT and partial-thickness holes may be distinguished from fullthickness holes (Figure 5).

\section{Discussion}

The IOLMaster 700 biometer includes a limited sweptsource optical coherence tomography scan of the entire length of the eye, including the central 1-mm of the fovea. Though the foveal scan is intended as a verification of ocular fixation during biometry acquisition, it potentially may be useful to cataract surgeons who do not routinely obtain OCT scans preoperatively to assess macular pathology. While the $22 \mu \mathrm{m}$ axial resolution and $1 \mathrm{~mm}$ horizontal scan length of IOLM SS-OCT significantly limits the image detail and tissue area that can be visualized compared with 1-6- $\mu \mathrm{m}$ axial resolution and 3-, 6- and 9-mm line scans of most commercial spectral-domain OCT and swept-source OCT systems, SS-OCT imaging in general

\section{A}
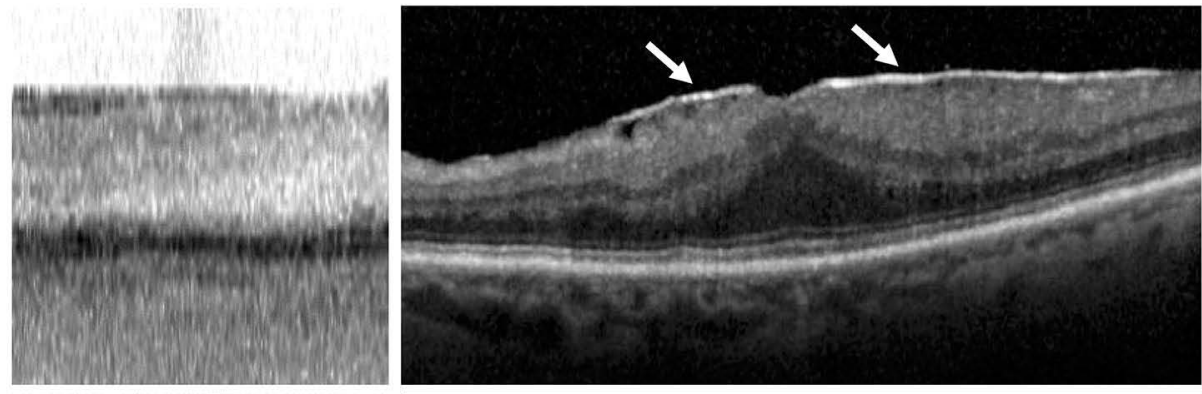

B
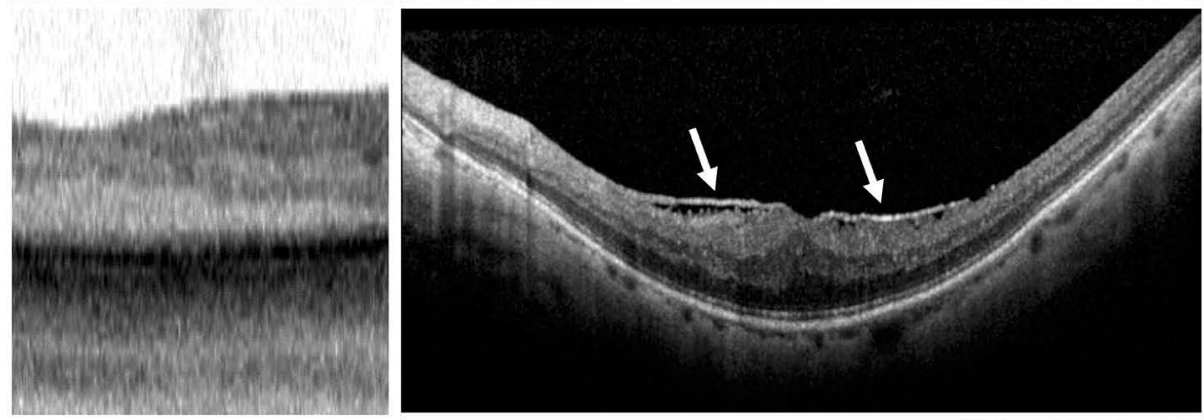

C
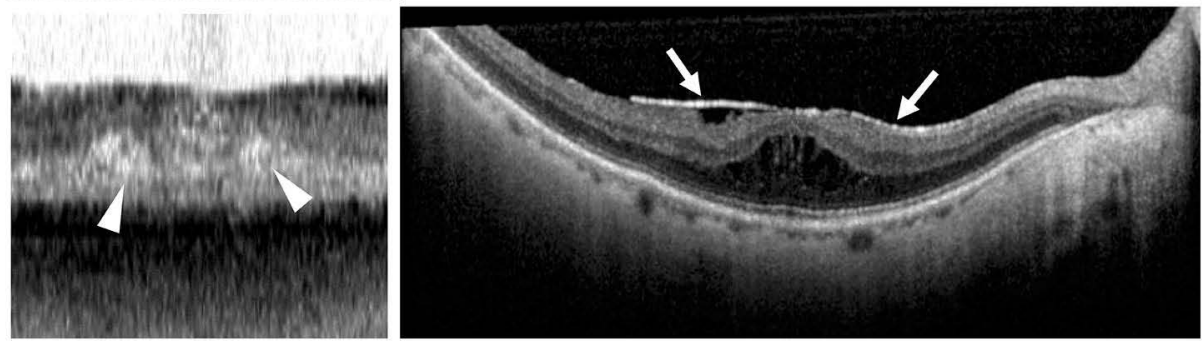

Figure 2 Epiretinal membrane appearance on swept-source OCT and spectral-domain OCT. (A-C, left) IOLMaster 700 SS-OCT scans of three different eyes with epiretinal membranes, demonstrating foveal thickening and intraretinal cystic spaces (arrowheads). (A-C, right) Corresponding Spectralis SD-OCT scans of epiretinal membranes (arrows) for comparison. 


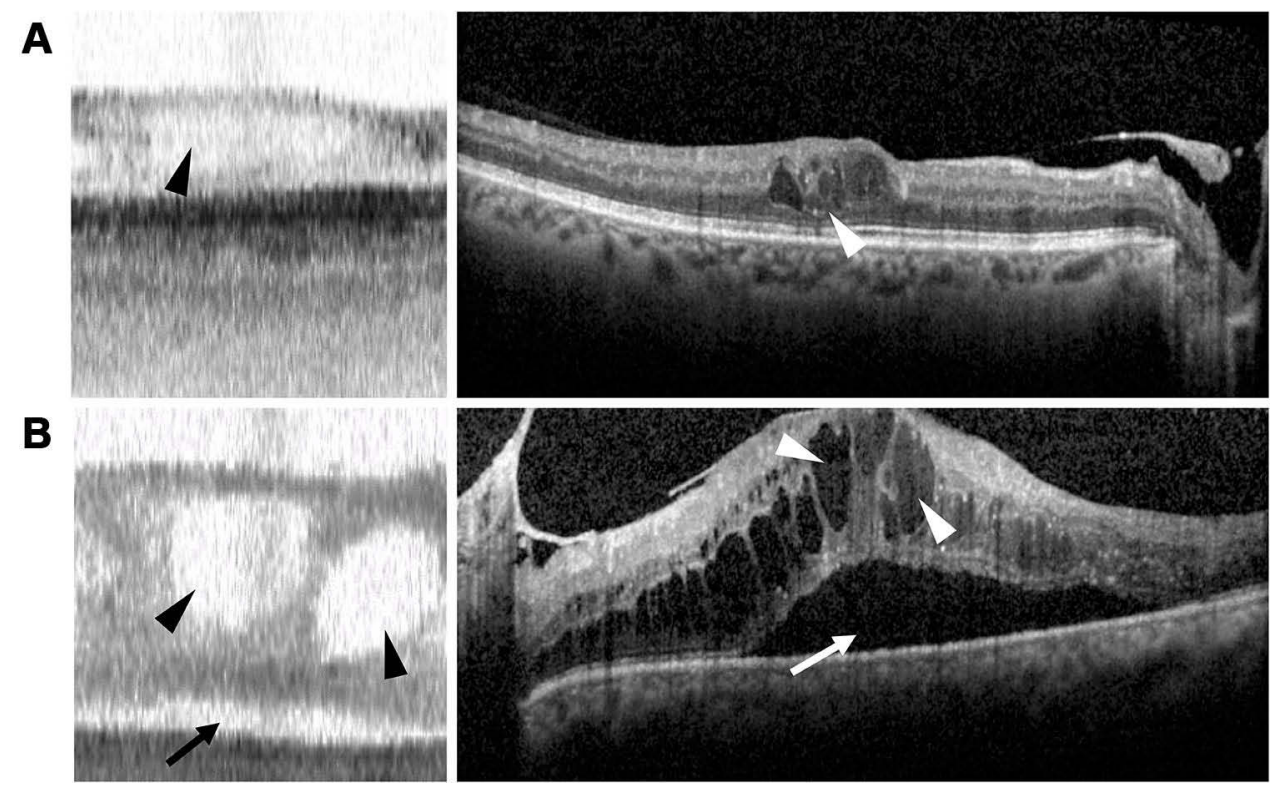

Figure 3 Macular edema appearance on swept-source OCT and spectral-domain OCT. (A and B, left) IOLMaster 700 SS-OCT scans of two different eyes with macular edema, demonstrating retinal thickening with intraretinal (black arrowheads) and subretinal fluid (black arrow). (A and B, right) Corresponding Spectralis SD-OCT scans of macular edema with intraretinal (white arrowheads) and subretinal (white arrow) fluid.

offers faster acquisition and greater penetration of deeper structures compared with SD-OCT. ${ }^{4-6}$

An abnormal-appearing IOLM SS-OCT was not uncommon in preoperative cataract surgery patients. In our study, $15.4 \%$ (179 eyes) of all preoperative cataract surgery eyes had an IOLM SS-OCT scan that was graded as abnormal. We found a sensitivity of $46.0 \%$ and a specificity of $83.2 \%$ of IOLM SS-OCT scans in detecting foveal pathology, using Spectralis SD-OCT scans as the gold standard for detecting presence or absence of pathology. The low sensitivity of the IOLM SS-OCT scan precludes it from being a reliable sole screening tool for detecting macular pathology in cataract surgery patients. The low sensitivity may be attributable to several reasons, including the low axial resolution of the IOLM 700's SSOCT, as well as the limited region (central 1-mm of the fovea) included in the scan, which excludes detection of any macular pathology outside of this area. In many instances, eyes with a normal-appearing SS-OCT were incorrectly graded as "normal" due to its corresponding SD-OCT scan revealing extrafoveal pathology, including epiretinal membranes involving the peripheral macula, drusen outside of the fovea, and non-central macular edema.

We also found a positive predictive value of $86.0 \%$ and a negative predictive value of $40.8 \%$ of IOLM SS-OCT in identifying retinal pathology. The relatively high positive predictive value and specificity suggest that an abnormal appearance of the fovea on IOLM SS-OCT should raise concern for presence of macular pathology and prompt further investigation prior to cataract surgery even if clinical examination was deemed unremarkable.

The most common pathology detected by IOLM SSOCT was epiretinal membrane, followed by macular degeneration, macular hole, macular edema, and other pathologies. For each pathologic condition, subtle but definitive differences exist between the appearance of the IOLM SS-OCT and SD-OCT images. Awareness that the color of the IOLM SS-OCT scan, which has a white background, is inverted from the color of the SD-OCT scan, which is set on a dark background, is an important detail for surgeons interpreting IOLM SS-OCT images to be familiar with. Consequently, hyporeflective areas that appear dark on SD-OCT (such as macular holes and cysts) appear bright on IOLM SS-OCT. Also, hyperreflective pathologic areas on SD-OCT (such as epiretinal membranes) are less distinct against the white background of the SS-OCT. Figures 2-5 provide a visual of the differences between the image as seen on the two devices and are discussed in more detail below.

The appearance of a wrinkled hyperreflective band above the nerve fiber layer, which helps identify epiretinal membranes on SD-OCT, is not usually visible on SSOCT. Surgeons should suspect an epiretinal membrane on 
A
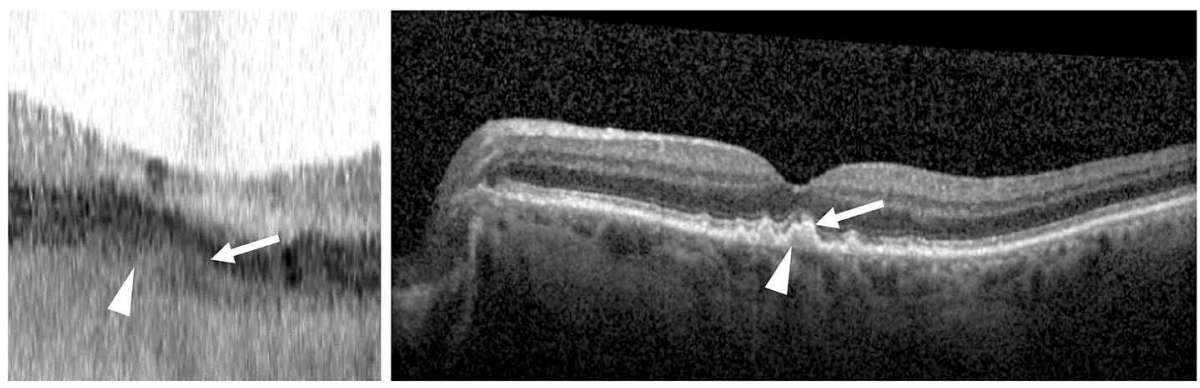

B
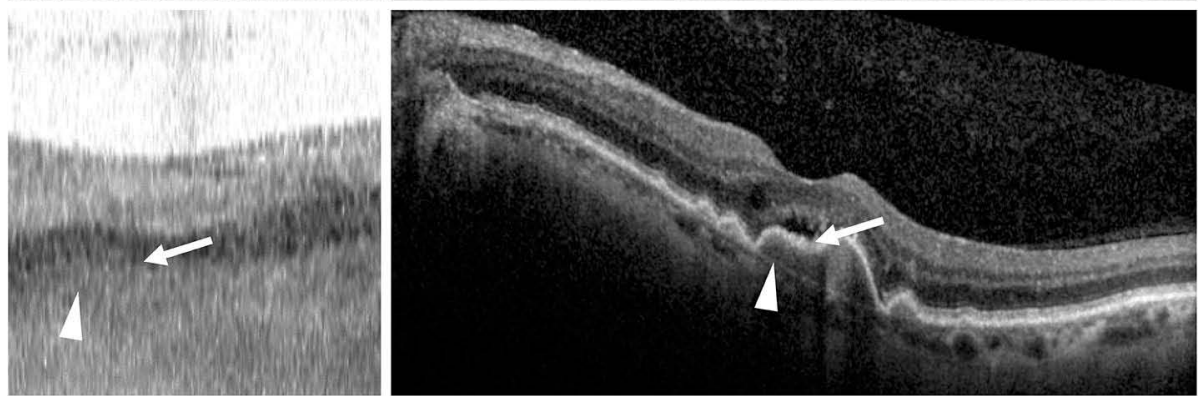

C
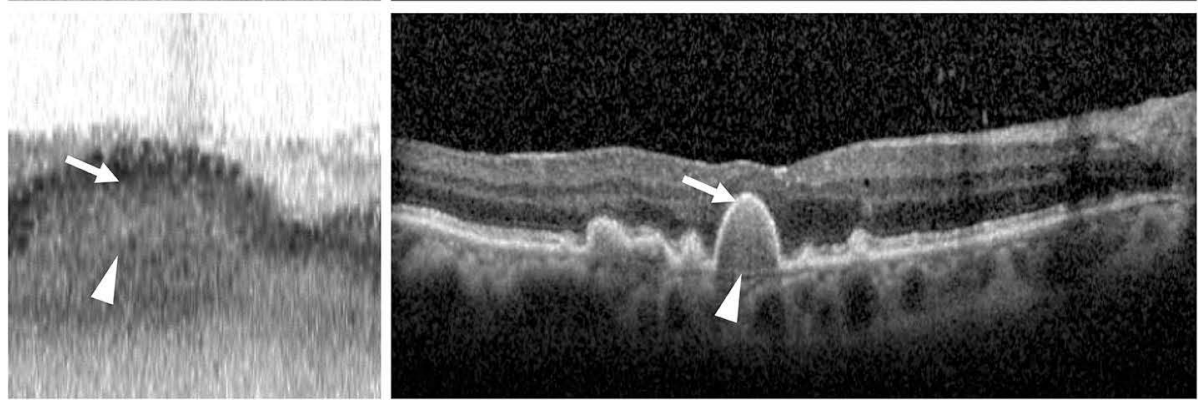

Figure 4 Drusen appearance on swept-source and spectral-domain OCT. (A-C, left) IOLMaster 700 SS-OCT scans of three different eyes with macular drusen (arrowheads) and retinal pigment epithelium (RPE) layer deformation (arrows). (A-C, right) Corresponding Spectralis SD-OCT scans of drusen (arrowheads) with RPE deformation (arrows).
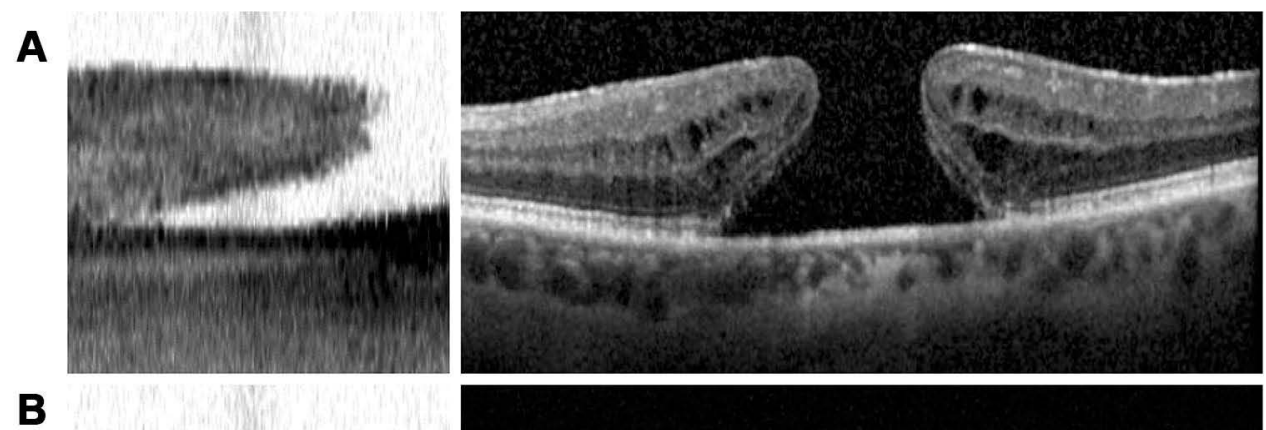

B
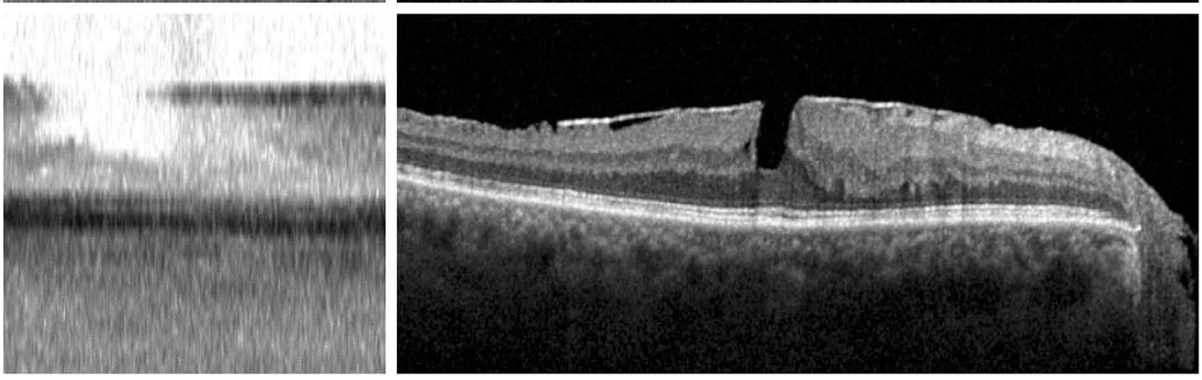

Figure 5 Macular hole appearance on swept-source and spectral-domain OCT. (A and B, left) IOLMaster 700 SS-OCT scans of two different eyes with macular holes. (A and B, right) Corresponding Spectralis SD-OCT scans of macular holes. 
SS-OCT if there is thickening of the fovea with straightening of the normal foveal pit. More advanced cases may show hyperreflective cysts in the outer retinal layers with associated retinal thickening. Poor ocular fixation can result in a similar thickened appearance with apparent loss or flattening of the foveal pit, but these scans can be distinguished by a relatively straight contour with retinal layers running in parallel, as opposed to a peaked appearance of the outer retinal layers from traction in true epiretinal membrane cases.

Smaller drusen tend to be difficult to detect on SS-OCT and may be recognized as an irregular contour of the retinal pigment epithelial layer, which appears as a dark band on SS-OCT. There were several eyes in which small drusen that appeared on Spectralis SD-OCT were not identifiable on the IOLM SS-OCT, including some that were in the peripheral macula and outside of the SSOCT scan field of view. Intraretinal and subretinal fluid is readily visualized on SS-OCT, and even small intraretinal cysts may be seen as bright punctate spots despite relatively low resolution. Most cases of central macular edema and macular holes were correctly identified on SSOCT.

Our findings are similar to a smaller study from Hirschall et al., in which IOLM SS-OCT scans were compared with RTVue SD-OCT scans (Optovue, Fremont, CA, USA) in 125 eyes, of which 65 had macular disease diagnosed previously on SD-OCT. ${ }^{7}$ They found sensitivity of IOLM SS-OCT ranging from $42-68 \%$ and specificity of 89-98\%, which is similar to our findings from a larger cohort. The most commonly identified macular pathologies in their study were epiretinal membrane, macular edema, and drusen. In another study by Tognetto et al. comparing the efficacy of IOLM SS-OCT scans against the Spectralis SD-OCT, the authors found a mean sensitivity of $81 \%$ and a mean specificity of $84 \%{ }^{8}$

Knowledge of the health of the macula is critical prior to cataract surgery, as it informs visual prognosis, identifies relative contraindications to using presbyopiacorrecting intraocular lenses, and may affect perioperative management. Subtle macular findings may be missed on fundoscopic biomicroscopy. In a study of 218 pre-cataract surgery eyes in which fundoscopic exam did not reveal any clinically evident macular pathology, SD-OCT identified macular abnormalities in 10 eyes (4.6\%), including epiretinal membrane, diabetic macular edema, and macular hole. ${ }^{9}$ In 5 of these eyes, cataract surgery was cancelled or postponed based on the
OCT findings. Another study similarly found $6.4 \%$ of preoperative eyes had macular pathology on OCT that was undetected on funduscopic exam, with epiretinal membrane being the most common. ${ }^{3}$ A third study of 265 eyes planned for cataract surgery with implantation of a multifocal or toric intraocular lens found macular pathology in 35 eyes (13.2\%) on SD-OCT, though this study did not exclude all eyes with known preexisting macular conditions. ${ }^{10}$ Yet despite the efficacy of OCT in detecting macular pathology that may be otherwise missed on clinical examination, the utility of obtaining routine preoperative OCTs in all eyes prior to cataract surgery is debatable given its added burden on cost and the negative impact of clinical load on imaging resources and personnel. There have been no studies examining the cost-effectiveness of routine OCTs prior to cataract surgery.

The findings of this study must be taken in context of several limitations. Since our study is a retrospective analysis, many patients included in the study did not have SSOCT and SD-OCT scans performed on the same visit. While only scans performed within 3 months of one another were included, and those eyes that had a procedural or surgical intervention (such as antivascular endothelial growth factor injection) performed in the interim were excluded, it is possible that certain pathologies such as macular edema may have evolved, regressed or developed new onset between when the SSOCT and SD-OCT scans were performed. Furthermore, whether or not a Spectralis SD-OCT had been obtained preoperatively was based on the clinical judgment of the attending ophthalmologist. Since a clinician may be more likely to obtain a Spectralis SD-OCT if there is a concern for macular pathology on clinical exam, the incidence of eyes with macular pathology on SD-OCT may be skewed higher. Comparing IOLM SS-OCT scans against this cohort could artifactually raise the sensitivity and positive predictive value of scans. On the other hand, some eyes with a normal-appearing IOLM SS-OCT had extrafoveal macular pathology, such as drusen, outside of the 1-mm scan zone. These scans would still be considered "false negative" scans when compared with Spectralis SD-OCT scans, which could artifactually lower the SS-OCT scan's sensitivity.

Despite these limitations, based on the high positive predictive value and specificity of an abnormal SS-OCT scan, we recommend that cataract surgeons using the IOLMaster 700 who do not routinely perform preoperative 
OCT incorporate a review of the SS-OCT scan included in the IOLM biometry data as part of their preoperative assessment of cataract surgery patients. Familiarity with the appearance of macular pathologies on IOLM SS-OCT is critical in identifying abnormal SS-OCT scans and is described earlier in our paper.

Our study findings suggest that an abnormal IOLMaster 700 SS-OCT scan of the fovea in an eye without known pathology has a high likelihood of being accurate and warrants further evaluation prior to cataract surgery. The low sensitivity of the IOLM SS-OCT scan in detecting macular pathology indicates that it alone is not a reliable screening tool to estimate foveal health. Since macular comorbidities are not uncommon in patients undergoing cataract surgery, we are in favor of incorporating SD-OCT as part of the preoperative evaluation for cataract surgery.

\section{Conclusion}

Our retrospective study found a high positive predictive value of an abnormal IOLMaster 700 SS-OCT scan and a high specificity of a normal SS-OCT scan. Comparison of abnormal SS-OCT scans to Spectralis SD-OCT demonstrated that common fovea-involving pathologies such as epiretinal membrane, drusen, macular holes and macular edema are identifiable by distinct morphologic features on the limited SS-OCT scan. Our paper characterizes these morphologic features. Based on the findings of our study, we withhold cataract surgery and recommend further evaluation of the retina for any preoperative cataract surgery patient with an abnormal IOLMaster 700 SS-OCT. Our study also found a low sensitivity of the IOLMaster SSOCT scan in detecting macular pathology, in part due to its low axial resolution and limited 1-mm scan length, which excludes detection of any extrafoveal pathology. Our study underscores the fact that one cannot rely on the limited SSOCT scan alone to exclude the possibility of macular pathology prior to cataract surgery. In practices where macular imaging is not routinely performed as part of the preoperative workup, surgeons should have a low threshold for performing SD-OCT.

\section{Abbreviations}

SS-OCT, swept-source optical coherence tomography; SDOCT, spectral-domain optical coherence tomography; IR, inner retinal; OR, outer retinal; BM, Bruch's membrane;
RPE, retinal pigment epithelium; PR, photoreceptor; $\mathrm{CH}$, choroid.

\section{Acknowledgments}

Supported in part by a grant from Research to Prevent Blindness (New York, NY) to the Department of Ophthalmology, Northwestern University Feinberg School of Medicine.

\section{Disclosure}

Dr Surendra Basti reports personal fees from JNJ vision, outside the submitted work. The authors report no other potential conflicts of interest for this work.

\section{References}

1. French DD, Margo CE, Behrens JJ, Greenberg PB. Rates of routine cataract surgery among medicare beneficiaries. JAMA Ophthalmol. 2017;135:163-165. doi:10.1001/jamaophthalmol.20 16.5174

2. Abdelmassih Y, El-Khoury S, Georges S, Guindolet D, Gabison E, Cochereau I. Preoperative spectral-domain optical coherence tomography in patients having cataract surgery. J Cataract Refract Surg. 2018;44(5):610-614. doi:10.1016/j.jcrs.2018.02.020

3. Enright NJ, Catran A, Perera N, Cheng Y, Adler P. Yield of routine pre-cataract surgery macular optical coherence tomography in finding clinically undetected macular pathology. Clin Exp Ophthalmol. 2017;45(8):829-831. doi:10.1111/ceo.12973

4. Copete S, Flores-Moreno I, Montero JA, Duker JS, Ruiz-Moreno JM Direct comparison of spectral-domain and swept-source OCT in the measurement of choroidal thickness in normal eyes. $\mathrm{Br}$ J Ophthalmol. 2014;98(3):334-338. doi:10.1136/bjophthalmol-2013303904

5. Tan CSH, Ngo WK, Cheong KX. Comparison of choroidal thicknesses using swept source and spectral domain optical coherence tomography in diseased and normal eyes. $\mathrm{Br} J$ Ophthalmol. 2015;99(3):354-358. doi:10.1136/bjophthalmol-2014-305331

6. Tan CS, Cheong KX, Lim LW, Sadda SR. Comparison of macular choroidal thicknesses from swept source and spectral domain optical coherence tomography. $\mathrm{Br} J$ Ophthalmol. 2016;100(7):995-999. doi:10.1136/bjophthalmol-2015-307541

7. Hirnschall N, Leisser C, Radda S, Maedel S, Findl O. Macular disease detection with a swept-source optical coherence tomography-based biometry device in patients scheduled for cataract surgery. J Cataract Refract Surg. 2016;42(4):530-536. doi:10.1016/j. jcrs.2016.02.029

8. Tognetto D, Pastore MR, De Giacinto C, et al. Swept-source optical coherence tomography biometer as screening strategy for macular disease in patients scheduled for cataract surgery. Sci Rep. 2019;9 (1):9912. doi:10.1038/s41598-019-46243-3

9. Creese K, Ong D, Zamir E. Should macular optical coherence tomography be part of routine preoperative cataract assessment? Clin Exp Ophthalmol. 2012;40(1):e118-e119. doi:10.1111/j.1442-9071.2011. 02623.x

10. Klein BR, Brown EN, Casden RS. Preoperative macular spectral-domain optical coherence tomography in patients considering advanced-technology intraocular lenses for cataract surgery. $J$ Cataract Refract Surg. 2016;42(4):537-541. doi:10.1016/j. jers.2016.01.036 


\section{Publish your work in this journal}

Clinical Ophthalmology is an international, peer-reviewed journal covering all subspecialties within ophthalmology. Key topics include: Optometry; Visual science; Pharmacology and drug therapy in eye diseases; Basic Sciences; Primary and Secondary eye care; Patient Safety and Quality of Care Improvements. This journal is indexed on PubMed

Submit your manuscript here: https://www.dovepress.com/clinical-ophthalmology-journal
Central and CAS, and is the official journal of The Society of Clinical Ophthalmology (SCO). The manuscript management system is completely online and includes a very quick and fair peer-review system, which is all easy to use. Visit http://www.dovepress.com/ testimonials.php to read real quotes from published authors. 